\title{
LOCAL TIMES AND SAMPLE FUNCTION PROPERTIES OF STATIONARY GAUSSIAN PROCESSES
}

\author{
BY \\ SIMEON M. BERMAN( $\left.{ }^{1}\right)$
}

1. Introduction. The theory of local times of a stochastic process was conceived in the work of Paul Levy on linear Brownian motion [9]. H. Trotter proved the first major theorem for the Brownian model [11]; and much has been discovered by many other authors, too numerous to list here. A survey of the theory and a bibliography are contained in the monograph of Ito and McKean [8]. Local times have apparently been studied and used for Markov processes only. In this paper, local times of another class of stochastic processes are examined-a class of stationary Gaussian processes. Extensions to other processes are also indicated.

In their recent monograph, Cramér and Leadbetter [6] summarized the current research for stationary Gaussian processes and their sample functions; their book is mostly about processes whose correlation functions are twice differentiable at the origin. These processes have absolutely continuous sample functions. The present paper is about a very different class: the correlation function $r(t)$ satisfies the following relation for $t \rightarrow 0: t^{2}(1-r(t)) \rightarrow \infty$. A typical example is: $1-r(t)$ is asymptotic to a constant multiple of $|t|^{\alpha}, 0<\alpha<2$. The sample functions, though continuous, are not only not absolutely continuous, but are nondifferentiable at almost every point; this can be inferred from the work of J. Yeh [12]. It is shown that such processes have local times with continuous sample functions, and then the connection between local times and first passage times are revealed. These results are used to establish the following peculiar property of the Gaussian process: The values crossed by the sample function finitely many times in an interval form a set of category 1 in the image of that interval. A new inequality for the probability of first passage is incidentally obtained in $\$ 6$.

The well-known dichotomy theorem of Beljaev [1] states that the sample functions of a separable stochastically continuous, stationary Gaussian process are either almost all continuous, or else almost all unbounded; furthermore, a sufficient condition for continuity is the boundedness of $|\log | t||^{a}(1-r(t)), t \rightarrow 0$, for some constant $a>1$. In $\S 8$, a result for the unbounded case is proven: If

$$
\liminf _{t \rightarrow 0}|\log | t||(1-r(t))
$$

Received by the editors February 26, 1968.

( $\left.{ }^{1}\right)$ This paper represents results obtained at the Courant Institute of Mathematical Sciences, New York University, under the sponsorship of the National Science Foundation Grant NSF-GP-7378, and NSF-GW-2049. 
is positive, then almost all the sample functions spend positive time in every set of positive Lebesgue measure. This result is shown to provide a probabilistic proof of a theorem of Carathéodory on measurable functions: There is a measurable function of a real variable such that the inverse image of every nonempty open interval intersects every nonempty open interval in a set of positive measure.

One novelty of the present work is the use of Fourier analysis in local time: In order to "differentiate" the occupation time, the derivative is computed by means of the inversion formula for the characteristic function.

The author is grateful to the referee for his constructive comments.

2. Occupation time distributions and local times of measurable functions. Let $f(t)$ be a real valued measurable function defined on the interval $0 \leqq t \leqq 1$, and define $D(x)$ as $D(x)=1$ if $x \leqq 0$, or $=0$ if $x>0$. D is a Borel measurable function so that the composite function of $(t, x), D(f(t)-x)$, is measurable in the pair $(t, x)$; hence, the integral $F(x)=\int_{0}^{1} D(f(t)-x) d t$ is well defined. The function $F(x)$ is a probability distribution function; indeed, if the unit interval with Lebesgue measure is considered as a probability space, and $f(\cdot)$ as a random variable, then $F$ is the distribution of $f(\cdot)$. For our purposes it is convenient to call $F$ the "occupation time distribution" of the function $f(t): F(x)$ is the "amount of time in $[0,1]$ spent by $f$ at values less than or equal to $x$."

For any real valued or complex valued Borel measurable function $g$, we have

$$
\int_{0}^{1} g(f(t)) d t=\int_{-\infty}^{\infty} g(x) d F(x)
$$

in the sense that if one of the two integrals exist so does the other and the two are equal; indeed, this follows by a standard "change of variable" of integration.

Formula (2.1) provides a very convenient expression for the characteristic function of $F$ :

$$
\int_{-\infty}^{\infty} \exp (i u x) d F(x)=\int_{0}^{1} \exp [i u f(t)] d t
$$

Definition 2.1. When $F(x)$ is absolutely continuous, its derivative, denoted by $\phi(x)$, is called the local time of $f(t)$. (By "derivative" we mean the Radon-Nikodym derivative.)

The characteristic function of $F$ is the main instrument used to probe the local time. The following lemma on characteristic functions is fundamental for our study:

Lemma 2.1. Let $F$ be a distribution function whose characteristic function is square-integrable; then $F$ is absolutely continuous and its derivative is square-integrable.

Proof. For every square-integrable function $g$ on the real line, let $\hat{g}$ be its Fourier transform. Define the linear functional $S(g), g \in L_{2}$, as

$$
S(g)=\int_{-\infty}^{\infty} \hat{g}(u) \hat{\phi}(-u) d u
$$


where $\hat{\phi}(u)$ is the characteristic function of $F$. The Cauch $y^{\prime}$-Schwarz inequality and the Parseval relation imply that

$$
|S(g)|^{2} \leqq \int_{-\infty}^{\infty}|g(x)|^{2} d x \cdot \int_{-\infty}^{\infty}|\hat{\phi}(u)|^{2} d u
$$

hence, $S$ is a bounded linear functional. By the representation theorem for such functionals there exists a function $\phi \in L_{2}$ such that

$$
S(g)=\int_{-\infty}^{\infty} g(x) \phi(x) d x .
$$

If $g$ is the indicator function of a finite interval $[a, b]$, then, by the Parseval relation,

$$
\int_{a}^{b} \phi(x) d x=\int_{-\infty}^{\infty} \frac{\exp (i u b)-\exp (i u a)}{2 \pi i u} h(-u) d u=S(g)
$$

By the inversion formula for characteristic functions, the middle member above is equal to $F(b)-F(a)$ at all points $a, b$ of continuity of $F$. The function $\phi$ is integrable over every finite interval because it is square-integrable; therefore, its indefinite integral is absolutely continuous. It follows from the equation above that $F(x)$ cannot have any discontinuities and that

$$
\int_{a}^{b} \phi(x) d x=F(b)-F(a) \text { for all } a, b
$$

thus, $\phi$ is the derivative of $F$.

This lemma has an immediate application to local time:

LEMMA 2.2. If

$$
\int_{-\infty}^{\infty}\left|\int_{0}^{1} \exp [i u f(t)] d t\right|^{2} d u<\infty,
$$

then $F$ is absolutely continuous and $\phi$ is square integrable. If

$$
\int_{-\infty}^{\infty}\left|\int_{0}^{1} \exp [\operatorname{iuf}(t)] d t\right| d u<\infty
$$

then, there is a version of $\phi$ that is continuous.

Proof. The first assertion is a consequence of Lemma 2.1 and formula (2.2); and the second is a consequence of the fact that a distribution function has a continuous derivative if the characteristic function is absolutely integrable [6, p. 94].

LEMMA 2.3. If $F$ is absolutely continuous and $\phi$ square integrable, then

$$
\left|\int_{0}^{1} g(f(t)) d t\right|^{2} \leqq \int_{-\infty}^{\infty}|g(x)|^{2} d x \cdot \int_{-\infty}^{\infty}|\phi(x)|^{2} d x,
$$

for any square-integrable, Borel measurable $g$. 
Proof. Under the hypothesis, the domain of validity of the equation (2.1) includes all square-integrable $g$ because the right-hand side of $(2.1)$ is

$$
\int_{-\infty}^{\infty} g(x) \phi(x) d x
$$

thus, the lemma follows by application of the Cauchy-Schwarz inequality.

The properties of the local time are related to those of the function $f$ : If the former is "smooth," then the latter does not "spend too much time" near any particular value in its range; for example, if $\phi$ is square-integrable, then, by Lemma 2.3,

$$
\begin{aligned}
\int_{0}^{1} \exp (-|f(t)-x|)|f(t)-x|^{-\alpha} d t & \\
& \leqq\left\{\int_{-\infty}^{+\infty} \exp (-2|y|)|y|^{-2 \alpha} d y\right\}^{1 / 2} \cdot\left\{\int_{-\infty}^{\infty}|\phi(y)|^{2} d y\right\}^{1 / 2}
\end{aligned}
$$

for all $x$, when $\alpha<1 / 2$. This implies that $f(t)$ cannot satisfy a local Hölder condition of order greater than 2 at any point of its domain. If $\phi$ is essentially bounded, then

$$
\left|\int_{0}^{1} g(f(t)) d t\right| \leqq \int_{-\infty}^{\infty}|g(x)| d x \cdot \text { es sup } \phi
$$

for any absolutely integrable $g$; in particular,

$$
\int_{0}^{1} \exp (-|f(t)-x|)|f(t)-x|^{-\alpha} d t \leqq \int_{-\infty}^{\infty} \exp (-|y|)|y|^{-\alpha} d y \cdot \operatorname{es~sup} \phi
$$

for all $x$, when $\alpha<1$. This implies that $f(t)$ cannot satisfy a local Hölder condition of order greater than 1 at any point of its domain.

The rest of this section contains material on the convergence of an approximating sequence of occupation time distributions; these results will be applied in $\S 7$. Let $f(t)$ be a continuous function with an absolutely continuous occupation time distribution $F(x)$ with derivative $\phi(x)$. Let $\left\{f_{n}(t), n=1,2, \ldots\right\}$ be the following sequence of functions approximating $f(t)$ on $[0,1]: f_{n}(t)$ is equal to $f(t)$ at all points of the form $t=k 2^{-n}, k=0,1, \ldots, 2^{n}$, and is linear between any two such successive points. The sequence $\left\{f_{n}\right\}$ converges uniformly to $f$. Let $F_{n}(x)$ be the occupation time distribution of $f_{n}$; then, $F_{n}$ converges weakly (even pointwise) to $F$; indeed, the characteristic function of $F_{n}$ converges to that of $F$ :

$$
\int_{0}^{1} \exp \left[i u f_{n}(t)\right] d t \rightarrow \int_{0}^{1} \exp [i u f(t)] d t,
$$

because $f_{n}$ converges to $f$.

We assume that for every $n \geqq 1$, no two of the values $f\left(k 2^{-n}\right), k=0,1, \ldots, 2^{n}$, are equal, that is, the approximating function is nowhere constant. For each $n>0$, $F_{n}$ is absolutely continuous. This is directly visible from the piecewise linearity of 
$f_{n}$; or, more formally, from the fact that the characteristic function of $F_{n}$ is a sum of expressions of the form

$$
\int_{(k-1) 2^{-n}}^{k 2-n} \exp (\text { iuct }) d t, \quad c=\text { constant }
$$

each of which is square-integrable, so that Lemma 2.2 implies absolute continuity. Let $\phi_{n}(x)$ be the local time of $f_{n}$; we now derive a formula for it. For each number $x$ which is not one of the values $f\left(k 2^{-n}\right), k=0,1, \ldots, 2^{n}$, define $\xi_{n k}(x)$ as

$$
\begin{aligned}
\xi_{n k}(x) & =1, \quad \text { if } f\left(k 2^{-n}\right)-x \text { and } f\left((k-1) 2^{-n}\right)-x \text { are of opposite sign, } \\
& =0, \quad \text { otherwise, } \quad k=0,1, \ldots, 2^{n}
\end{aligned}
$$

in other words, $\xi_{n k}(x)$ is 1 if $f$ crosses the level $x$ somewhere in the open interval $\left((k-1) 2^{-n}, k 2^{-n}\right)$, and 0 if not. Put $f_{n k}=f\left(k 2^{-n}\right)-f\left((k-1) 2^{-n}\right)$.

LEMMA 2.4. $\phi_{n}(x)$ is equal to

$$
2^{-n} \sum_{k=1}^{2^{n}} \xi_{n k}(x) /\left|f_{n k}\right|
$$

at all points $x$ not equal to one of the values $f\left(k 2^{-n}\right), k=0,1, \ldots, 2^{n}$. (It may be arbitrarily defined at the latter values because the derivative is unique almost everywhere.)

Proof. Let $x$ be a value not equal to any of the specified ones. If it is larger than $\max _{k} f\left(k 2^{-n}\right)$ or smaller than $\min _{k} f\left(k 2^{-n}\right)$, put $\phi_{n}(x)=0$; otherwise, there is a finite set of points $\tau_{1}, \tau_{2}, \ldots$ in $(0,1)$ such that $f\left(\tau_{j}\right)=x$, and $\tau_{j}$ lies in some open interval $\left((k-1) 2^{-n}, k 2^{-n}\right), j=1,2, \ldots$. The function $f_{n}$ is linear as it passes through the level $x$ at the point $\tau_{j}$, and has slope equal to $2^{n} f_{n k}$; thus, the Lebesgue measure of the $t$-set for which $\left|f_{n}(t)-x\right|<\varepsilon$ is equal to $\varepsilon$ times the sum of the reciprocals of the absolute values of the slope at $\tau_{1}, \tau_{2}, \ldots$ if $\varepsilon>0$ is sufficiently small. None of these slopes is 0 because we have assumed that $x$ is not one of the numbers $f\left(k 2^{-n}\right)$. It follows that (2.3) is the formula for the derivative of $F_{n}$ at $x$.

The weak convergence of $F_{n}$ to $F$ and the continuity of $F$ imply that

$$
\int_{I} \phi_{n}(x) d x \rightarrow \int_{I} \phi(x) d x
$$

for every interval $I$. This does not imply that $\phi_{n}$ converges to $\phi(x)$, or even has a limit; nevertheless, the relation (2.4) does give information about the relation between $\phi_{n}$ and $\phi$.

LEMma 2.5. For any interial $[a, b]$, the maximal term of the sum

$$
\sum_{k=1}^{2^{n}} 2^{-n} \int_{a}^{b} \frac{\xi_{n k}(x)}{\left|f_{n k}\right|} d x
$$

(which is the integral of $\phi_{n}$ over $[a, b]$ ) tends to 0 as $n$ becomes infinite. 
Proof. Put $c=(k-1) 2^{-n}$ and $d=k 2^{-n}$. The function $\xi_{n k}(x)$ is, by definition, the function

$$
\begin{aligned}
\xi_{n k}(x) & =1 & & \text { if } f(c)<x<f(d), \\
& =1 & & \text { if } f(d)<x<f(c), \\
& =0 & & \text { elsewhere }
\end{aligned}
$$

therefore, its integral over $[a, b]$ is

$$
\begin{array}{rlr}
\int_{a}^{b} \xi_{n k}(x) d x & =\min (b, f(d))-\max (a, f(c)) & \text { if } f(c)<f(d), \\
& =\min (b, f(c))-\max (a, f(d)) & \text { if } f(d)<f(c)
\end{array}
$$

thus, in either of the latter cases, the integral $\int_{a}^{b}\left(\xi_{n k}(x) /\left|f_{n k}\right|\right) d x$ is at most 1 ; hence, each term in the sum (2.5) is bounded by $2^{-n}$.

LEMMA 2.6. Let $f$ be a continuous function on a closed interval, with a continuous local time $\phi$; then the set of zeros of $\phi$ is nowhere dense in the range of $f$.

Proof. Let the closed interval $I$ be the range of $f$. The set of zeros of $\phi$ is closed because $\phi$ is continuous. This set contains no nonempty open subintervals of $I$; indeed, if $\phi$ would vanish throughout such a subinterval $J$, the (open) set $f^{-1}(J)$ would have measure 0 , and be, therefore, empty; however, this would contradict the connectedness of the range of $f$.

For each $x$, the sum $\sum_{k=1}^{2^{n}} \xi_{n k}(x)$ is the number of times that the approximating function $f_{n}(t)$ crosses the level $x$. The sequence of these sums is monotonically nondecreasing and converges to a limit $N(x)$ which may be either finite or infinite. We note that $N(x)$ is at least equal to the number of times that $f$ "crosses" the value $x$ : If in every neighborhood of a point $t$ there are dyadic rational numbers $c, d: c<t<d$ such that $f(c)-x$ and $f(d)-x$ are of opposite sign, then $f$ crosses $x$ at $t$ (cf. [13]).

THEOREM 2.1. Let f be a continuous function on a closed interval with a continuous local time $\phi$. Suppose that each approximating function $f_{n}$ is nowhere constant. The set of values $x$ where $N(x)$ is finite is of category 1 in the range of $f$.

Proof. For a fixed positive integer $m$, the set $\{x: N(x)>m\}$ is an open set. If $N(x)$ exceeds $m$, then there is an integer $n$ sufficiently large so that the sum $\sum_{k=1}^{2^{n}} \xi_{n k}(x)$ exceeds $m$, that is, the "broken line" $f_{n}$ crosses $x$ more than $m$ times. It follows that this broken line must cross every value in some neighborhood of $x$ more than $m$ times; indeed, the raising or lowering of the level $x$ by a sufficiently small amount will not decrease the number of crossings. It follows that $N(y)$ exceeds $m$ for all values of $y$ sufficiently near $x$.

We conclude that $\{x: N(x) \leqq m\}$ is closed.

Now we show that $\{x: N(x) \leqq m\}$ contains no nonempty open subintervals of the range of $f$. If $(a, b)$ is a nonempty open subinterval on which $N(x) \leqq m$, then the sum in (2.5) contains at most $m$ terms; thus, by Lemma 2.5 , the integral of $\phi_{n}$ over 
$(a, b)$ converges to 0 . From the relation (2.4) it follows that the integral of $\phi$ over $(a, b)$ is 0 ; therefore, by the continuity of $\phi, \phi$ must vanish throughout $(a, b)$. It now follows from Lemma 2.6 that $(a, b)$ is not in the range of $f$.

We conclude that the set $\{x: N(x) \leqq m\}$ is nowhere dense in the range of $f$ because it has a closed intersection with the range and no interior points in it. It follows that the set upon which $N(x)$ is finite is of category 1 in the range of $f$ because it is the union of the sets $\{x: N(x) \leqq m\}, m \geqq 1$.

3. Local times for stochastic processes. Let $(\Omega, \mathscr{F}, P)$ be a probability space and $X(t, \omega), 0 \leqq t \leqq 1, \omega \in \Omega$, a separable stochastic process measurable in the pair $(t, \omega)$, that is, with respect to the product sigma-field of $\mathscr{F}$ and the Lebesgue measurable subsets of $[0,1]$. For each pair $(x, \omega)$, we define the occupation time distribution of the function $X(\cdot, \omega)$ :

$$
F(x, \omega)=\int_{0}^{1} D(X(t, \omega)-x) d t .
$$

For almost every $\omega, F(\cdot, \omega)$ is a distribution function. If it is absolutely continuous with derivative $\phi(\cdot, \omega)$, then the latter is called the local time of $X(t, \omega), 0 \leqq t \leqq 1$.

Let $E$ be the expectation operator corresponding to the probability measure $P$. When a function of $(t, \omega)$ or $(x, \omega)$ appears under the expectation or probability signs, the argument $\omega$ will be omitted; for example. we shall write $E X(t, \omega)$ as $E X(t)$.

The results of $\S 2$ on occupation time distributions for measurable functions can be extended to the stochastic process $X(t, \omega)$.

Lemma 3.1. If

$$
\int_{-\infty}^{\infty}\left|\int_{0}^{1} \int_{0}^{1} E \exp [i u(X(t)-X(s))] d s d t\right| d u<\infty,
$$

then, for almost all $\omega, F(\cdot, \omega)$ is absolutely continuous and $\phi(\cdot, \omega)$ is square-integrable.

Proof. The hypothesis implies that $E\left(\int_{-\infty}^{\infty}\left|\int_{0}^{1} \exp [i u X(t)] d t\right|^{2} d u\right)<\infty$; hence, for almost all $\omega$, the characteristic function of $F(\cdot, \omega), \int_{0}^{1} \exp [i u X(t, \omega)] d t$, is square-integrable in $u$. The assertion of the lemma now follows from the first part of Lemma 2.2.

LEMMA 3.2. If for every $s, t, 0 \leqq s, t \leqq 1$.

$$
\int_{-\infty}^{\infty}|E \exp [i u(X(t)-X(s))]| d u<\infty,
$$

then the probability density function $p(x ; t, s)$ of the random tariable $X(t, \cdot)-X(s, \cdot)$ exists and is continuous, and the hypothesis of Lemma 3.1 is then equiralent to

$$
\int_{0}^{1} \int_{0}^{1} p(0 ; s, t) d s d t<\infty
$$


Proof. The first assertion is the well-known result that a distribution function is continuously differentiable if the characteristic function is absolutely integrable [4, p. 94]. The second assertion follows by application of Fubini's theorem and the inversion formula for the density as an integral of the characteristic function.

LEMMA 3.3. If, for some $\varepsilon>0$,

$$
\int_{-\infty}^{\infty}\left|\int_{0}^{1} \int_{0}^{1} E \exp [i u(X(t)-X(s))] d s d t\right||u|^{1+\varepsilon} d u<\infty
$$

then, for almost all $\omega, F(\cdot, \omega)$ is absolutely continuous and $\phi(\cdot, \omega)$ is continuous.

Proof. The hypothesis implies that

$$
\int_{-\infty}^{\infty} E\left|\int_{0}^{1} \exp [i u X(t)] d t\right|^{2}|u|^{1+\varepsilon} d u<\infty
$$

which implies that

$$
\int_{-\infty}^{\infty}|u|^{1+\varepsilon}\left|\int_{0}^{1} \exp [i u X(t, \omega)] d t\right|^{2} d u<\infty
$$

for almost all $\omega$; this, by the Cauchy-Schwarz inequality, implies that

$$
\int_{-\infty}^{\infty}\left|\int_{0}^{1} \exp [i u X(t, \omega)] d t\right| d u<\infty
$$

for almost all $\omega$. The assertion of the lemma now follows from the second part of Lemma 2.2.

EXAMPLE 3.1. Let $X(t, \omega)$ be a process with stationary independent increments and with characteristic function

$$
E \exp [i u(X(t)-X(s))]=\exp [-(t-s) \lambda(u)] .
$$

The hypothesis of Lemma 3.1 is fulfilled if the function $\left(1-e^{-\lambda(u)}\right) / \lambda(u)$ is absolutely integrable. The hypothesis of Lemma 3.3 is fulfilled if the modulus of that function times $|u|^{1+\varepsilon}$ is integrable. In this example, the results obtained are weaker than those already known [2].

EXAMPLE 3.2. Let $X(t, \omega)$ be a Gaussian process with covariance function $R(s, t)=E X(s) X(t)-E X(s) \cdot E X(t)$. The random variable $X(t, \cdot)-X(s, \cdot)$ has a Gaussian distribution with mean $E X(t)-E X(s)$ and variance $R(s, s)+R(t, t)-$ $2 R(s, t)$. The hypothesis of Lemma 3.2 is satisfied; thus, a sufficient condition for the hypothesis of Lemma 3.1 to be satisfied is

$$
\int_{0}^{1} \int_{0}^{1}(R(t, t)+R(s, s)-2 R(s, t))^{-1 / 2} d s d t<\infty .
$$


EXAMPLe 3.3. Let us specialize the previous example to the case of a stationary Gaussian process, normalized so that $E X(t) \equiv 0$ and $E X^{2}(t) \equiv 1$; put $r(s-t)=$ $R(s, t)$. The condition (3.1) is equivalent to

$$
\int_{0}^{1}(1-r(t))^{-1 / 2}(1-t) d t<\infty
$$

Suppose that $1-r(t)$ vanishes only for $t=0$; then the condition (3.2) is satisfied if $1-r(t)$ approaches 0 more slowly than a constant multiple of $|t|^{\alpha}, t \rightarrow 0$, for some $\alpha, 0<\alpha<2 ;(3.2)$ is certainly not fulfilled if $r^{\prime \prime}(0)$ exists. (Here we are assuming that the process is real-valued so that $r^{\prime}(0)=0$.)

Our first result on the continuity of the local time of the stationary Gaussian process in Example 3.3 is:

THEOREM 3.1. If for some $\varepsilon>0$,

$$
\int_{0}^{1}(1-r(t))^{-1-\varepsilon} d t<\infty
$$

then, for almost all $\omega, F(\cdot, \omega)$ is absolutely continuous and $\phi(\cdot, \omega)$ is continuous.

Proof. The integral in the hypothesis of Lemma 3.3 takes the particular form

$$
\int_{-\infty}^{\infty}\left(\int_{0}^{1} \int_{0}^{1} \exp \left[-u^{2}(1-r(s-t))\right] d s d t\right)|u|^{1+\varepsilon} d u
$$

Interchange the order of integration: By integrating first with respect to $u$, we find that the finiteness of the above multiple integral is identical with the finiteness of $\int_{0}^{1} \int_{0}^{1}(1-r(s-t))^{-1-(\varepsilon / 2)} d s d t$, implied by condition (3.3).

Theorem 3.1 shows that the local time is continuous when $1-r(t)$ is bounded below by a constant multiple of $|t|^{\alpha}$ as $t \rightarrow 0$ for some $\alpha, 0<\alpha<1$; however, the theorem is not strong enough to cover $\alpha, 1 \leqq \alpha<2$, to be treated in the next two sections by more elaborate methods.

4. The local time process and the continuity of its sample functions. Suppose that $X(t, \omega)$ is the process defined in $\S 3$, and that $F(x, \omega)$ is absolutely continuous in $x$ with derivative $\phi(x, \omega)$ for almost all $\omega$. We would like to consider the function $\phi(x, \omega)$ as a stochastic process on $(\Omega, \mathscr{F}, P)$ with the time parameter $x,-\infty<x<\infty$; then prove that a separable version of this process has continuous sample functions; and conclude that $\phi(x, \omega)$ is continuous in $x$ for almost all $\omega$. There are two gaps in this argument: For each $\omega$, the function $\phi(x, \omega)$ is defined for almost every $x$, not for every $x$, so that $\phi(x, \cdot)$ is not a well-defined random variable for each $x$; and the fact that a separable version of the derived process has continuous sample functions does not necessarily imply that the original function $\phi(x, \omega)$ is continuous in $x$ for almost all $\omega$. 
Here are the steps we shall take in filling these gaps and proving that $\phi(\cdot, \omega)$ is continuous for almost all $\omega$ :

1. Construct a stochastic process $\psi(x, \omega),-\infty<x<\infty$, on $(\Omega, \mathscr{F}, P)$, which, without loss of generality, may be assumed separable.

2. Prove that if every separable version of $\psi(x, \omega)$ is $(x, \omega)$-measurable, then any such version serves as the derivative of $F$.

3 . Note that if every separable version of $\psi(x, \omega)$ has continuous sample functions, then every such version is also measurable, and serves as a (continuous) derivative of $F$.

4. Find conditions on the finite-dimensional distributions of the process $X$ which are sufficient for the continuity of the sample functions of a separable version of the process constructed in step 1 .

Let $X(t, \omega)$ satisfy the condition

$$
\int_{-\infty}^{\infty} \int_{-\infty}^{\infty} \int_{0}^{1} \int_{0}^{1}|E \exp [i u X(s)+i v X(t)]| d s d t d u d v<\infty
$$

In the case of the Gaussian process described in Example 3.2, the condition (4.1) is equivalent, by the inversion formula for characteristic functions, to

$$
\int_{0}^{1} \int_{0}^{1}\left[R(s, s) R(t, t)-R^{2}(s, t)\right]^{-1 / 2} d s d t<\infty
$$

In the particular case of the stationary Gaussian process in Example 3.3, the condition (4.1) is identical with (3.2) because of the relation $1-r^{2} \sim 2(1-r), r \rightarrow 1$.

In order to construct a stochastic process $\psi(x, \omega),-\infty<x<\infty$, to serve as a local time "candidate," we write a formal expression for the density of $F(x, \omega)$ in terms of the characteristic function inversion formula, and then show that the formula has validity in the required sense.

Theorem 4.1. Put

$$
\psi_{N}(x, \omega)=\frac{1}{2 \pi} \int_{-N}^{N} \exp (-i u x) \int_{0}^{1} \exp [i u X(t, \omega)] d t d u, \quad N=1,2, \ldots
$$

If (4.1) holds, then, for each $x$, there exists a random tariable $\psi(x, \omega)$ such that

$$
\lim _{x \rightarrow \infty} E\left|\psi_{N}(x)-\psi(x)\right|^{2}=0 \text { uniformly in } x:
$$

furthermore, the stochastic process $\psi(x, \omega),-\infty<x<\infty$, may be assumed to be separable.

Proof. If $N>M$, then

$$
\psi_{v}(x, \omega)-\psi_{M}(. x, \omega)=\frac{1}{2 \pi}\left(\int_{-N}^{-M}+\int_{M}^{N}\right) \exp (-i u . x) \int_{0}^{1} \exp [i u X(t, \omega)] d t d u:
$$


thus, by virtue of the inequality $|x+y|^{2} \leqq 2|x|^{2}+2|y|^{2}$ for complex $x$ and $y$, $E\left|\psi_{N}(x)-\psi_{M}(x)\right|^{2}$ is dominated by

$$
\begin{aligned}
2 E\left|\frac{1}{2 \pi} \int_{-N}^{-M} \exp (-i u x) \int_{0}^{1} \exp [i u X(t)] d t d u\right|^{2} \\
+2 E\left|\frac{1}{2 \pi} \int_{M}^{N} \exp (-i u x) \int_{0}^{1} \exp [i u X(t)] d t d u\right|^{2} .
\end{aligned}
$$

The first expression above is dominated by

$$
\frac{2}{(2 \pi)^{2}} \int_{-N}^{-M} \int_{-N}^{-M} \int_{0}^{1} \int_{0}^{1}|E \exp [i u X(s)-i v X(t)]| d s d t d u d v
$$

and the second is dominated by a similar one with integration over $u$ and $v$ from $M$ to $N$. By the hypothesis (4.1) each of these expressions converges to 0 (uniformly in $x$ ) as $M, N \rightarrow \infty$. The conclusion (4.2) now follows from the uniform convergence of $E\left|\psi_{N}(x)-\psi_{M}(x)\right|^{2}$ to 0 .

By the fundamental separability theorem [7, p. 57] there is a separable stochastic process $\tilde{\psi}(x, \omega),-\infty<x<\infty$, on $(\Omega, \mathscr{F}, P)$ such that for each $x, \tilde{\psi}(x, \omega)=\psi(x, \omega)$ for almost all $\omega$. The relation (4.2) remains valid if $\tilde{\psi}$ is substituted for $\psi$; hence, the second assertion of the theorem holds.

THEOREM 4.2. If the process $\psi(x, \omega)$ is measurable in $(x, \omega)$, then, for almost every $\omega, \psi(x, \omega)$ is absolutely integrable over every finite $x$-interval, and

$$
F(b, \omega)-F(a, \omega)=\int_{a}^{b} \psi(x, \omega) d x
$$

for every pair of real numbers $a, b$.

Proof. For each $a$ and $b$, the integral $\int_{a}^{b} \psi_{N}(x, \omega) d x$ is well defined and

$$
\lim _{N \rightarrow \infty} \int_{a}^{b} \psi_{N}(x, \omega) d x=F(b, \omega)-F(a, \omega)
$$

for almost every $\omega$; indeed, the existence of the integral follows from the continuity in $x$ of $\psi_{N}(x, \omega)$, and the convergence (4.4) is a consequence of the inversion formula for characteristic functions and the (absolute) continuity of $F(x, \omega)$ at every $x$ (in particular at $x=a, b)$.

The function $\psi_{N}(x, \omega)$ is not only continuous in $x$ but also bounded in $(x, \omega)$ (by $N / \pi)$; therefore, $E\left|\psi_{N}(x)\right|^{2}$ is a continuous function of $x$. Equation (4.2) implies that $E|\psi(x)|^{2}$ is finite for all $x$ and is the uniform (in $x$ ) limit of the sequence of continuous functions $\left\{E\left|\psi_{N}(x)\right|^{2}\right\}$; therefore, $E|\psi(x)|^{2}$ is continuous in $x$; consequently, it is integrable over every finite interval. Fubini's theorem now implies that $\psi(\cdot, \omega)$ is square-integrable over each finite interval for almost all $\omega$; therefore, it is absolutely integrable over each finite interval for almost all $\omega$; finally, for almost all $\omega$, it is absolutely integrable over every finite interval. 
Now we show that for each $a, b$, the equation (4.3) holds for almost all $\omega$. The absolute difference between the members on each side of the equation is dominated, for each $N$, by

$$
\left|F(b, \omega)-F(a, \omega)-\int_{a}^{b} \psi_{N}(x, \omega) d x\right|+\int_{a}^{b}\left|\psi(x, \omega)-\psi_{v}(x, \omega)\right| d x .
$$

By the result (4.4), the first term above converges to 0 as $N \rightarrow \infty$ for almost all $\omega$. The second term converges to 0 in mean square as $N \rightarrow \infty$; indeed, by the CauchySchwarz inequality, its expected square is dominated by the square root of

$$
|b-a| \int_{a}^{b} E\left|\psi(x)-\psi_{N}(x)\right|^{2} d x
$$

which, by (4.2), converges to 0 as $N \rightarrow \infty$.

We still have to show that for almost all $\omega$, the equation (4.3) holds for all $a, b$. By what has just been shown in the preceding paragraph and by Fubini's theorem, for almost all $\omega$, the equation (4.3) holds for almost all $a$ and $b$. Now, on one hand, for almost all $\omega, \psi(\cdot, \omega)$ is absolutely integrable over every finite interval, so that its indefinite integral is an absolutely continuous function; and, on the other hand, $F(\cdot, \omega)$ is, by hypothesis, absolutely continuous for almost all $\omega$; hence, for almost all $\omega$, each function on either side of the equation (4.3) is determined for every $a$ and $b$ if it is determined for almost every $a$ and $b$. It follows that, for almost all $\omega$, the equation (4.3) holds for every $a$ and $b$.

THEOREM 4.3. If the process $\psi(x, \omega)$ is continuous in $x$ for almost all $\omega$, then $\psi(\cdot, \omega)$ serves as the (continuous) derivative of $F(\cdot, \omega)$ for almost all $\omega$.

Proof. The process $\psi(x, \omega)$ is $(x, \omega)$-measurable because it is separable and has continuous sample functions [7, p. 60]; therefore, by Theorem $4.2, \psi(\cdot, \omega)$ is the derivative of $F(\cdot, \omega)$ for almost all $\omega$.

Now we give a condition under which the sample functions of a separable version of $\psi$ are continuous. The condition of Kolmogoroff for the continuity of the sample functions of a separable process $U(x)$ is: there are positive constants $A, k$, and $\varepsilon$ such that

$$
E|U(x+h)-U(x)|^{k} \leqq A|h|^{1+\varepsilon}
$$

for all $h$ and $x$. Putting $U=\psi$ and $k=2 m$, we have the expression for the $2 m$ th absolute moment of $\psi(x+h, \cdot)-\psi(x, \cdot)$ :

$$
\begin{aligned}
E|\psi(x+h)-\psi(x)|^{2 m} & (2 \pi)^{-2 m} \lim _{N \rightarrow \infty} E \int_{-N}^{N} \cdots \int_{-N}^{N} \int_{0}^{1} \cdots \int_{0}^{1} \prod_{j=1}^{m}\left(\exp \left[-i u_{j}(x+h)\right]-\exp \left[-i u_{j} x\right]\right) \\
& \cdot\left(\exp \left[i v_{j}(x+h)\right]-\exp \left[i v_{j} x\right]\right) \\
& \quad \prod_{j=1}^{m} \exp \left[i u_{j} X\left(s_{j}\right)-i v_{j} X\left(t_{j}\right)\right] \prod_{j=1}^{m} d s_{j} d t_{j} d u_{j} d v_{j},
\end{aligned}
$$


where $s_{j}$ and $t_{j}$ are integrated from 0 to 1 , and $u_{j}$ and $v_{j}$ from $-N$ to $+N$, $j=1, \ldots, m$.

The entire discussion depends in no essential way on the choice of the unit interval as the domain of the stochastic process $X(t, \omega)$; the results are valid for an arbitrary interval on the real line: one has only to modify the domain of integration in (4.1) and in the subsequent integrals.

5. Continuity of the local time of certain processes with $1 \leqq \alpha<2$. Let $X(t, \omega)$ be a real valued separable measurable stationary Gaussian process with $E X(t) \equiv 0$ and $E X^{2}(t) \equiv 1$, and with covariance $E X(s) X(t)=r(s-t)$. Let $r(t)$ be continuous with the spectral form

$$
r(t)=\int_{-\infty}^{\infty} \exp (i u t) d G(u)
$$

where the spectral distribution $G$ is symmetric about $u=0$. In contrast to $\S 3$, where the assumptions were imposed on the covariance, the pending hypotheses are more conveniently put on the spectral function. Our first assumption is that the latter has an absolutely continuous component; this implies that there is no linear relationship among finitely many random variables of the process. Our second assumption is about the asymptotic behavior of $G$ for large values. Let $\alpha$ satisfy $1 \leqq \alpha<2$; and let $c$ be the reciprocal of the constant $\int_{-\infty}^{\infty} d u /\left(1+|u|^{1+\alpha}\right)$; then we suppose that

$$
\int_{-\infty}^{\infty} u^{2} d\left[\left|G(u)-c \int_{-\infty}^{u} d v /\left(1+|v|^{1+\alpha}\right)\right|\right]<\infty
$$

where the Stieltjes integral is taken with respect to the absolute variation of the difference between the two monotone functions. This condition is satisfied, in particular, if $G$ is differentiable, and

$$
G^{\prime}(u)=|u|^{-1-\alpha}+O\left(|u|^{3+\delta}\right) \quad(|u| \rightarrow \infty)
$$

for some $\delta>0$.

Now we analyze some properties of the particular covariance function

$$
r(t)=c \int_{-\infty}^{\infty} \exp (i u t) d u /\left(1+|u|^{1+\alpha}\right) .
$$

For $t \rightarrow 0$

$$
2(1-r(t)) \sim|t|^{\alpha} c \int_{-\infty}^{\infty}|1-\exp (i u)|^{2}|u|^{-1-\alpha} d u .
$$

(Proof. The left-hand side is equal to

$$
c \int_{-\infty}^{\infty}|1-\exp (i u t)|^{2} d u /\left(1+|u|^{1+\alpha}\right)
$$

now change the variable: $u \rightarrow u / t$.) 
For all $t, h$ and all $\beta<1$

$$
|r(t+h)-r(t)| \leqq 2 h^{\beta} c \int_{-\infty}^{\infty}|u|^{\beta} d u /\left(1+|u|^{1+\alpha}\right)
$$

(Proof. Apply the inequality $\left|e^{i x}-e^{i y}\right| \leqq 2|x-y|^{\beta}$ to the integrand of the integral form of $r(t+h)-r(t)$.)

For all $t, h, l$ and all $\beta<1$

$$
\begin{aligned}
\mid r(t+h+l)-r(t+h)-r(t+l) & +r(t) \mid \\
& \leqq|h|^{\beta}|l|^{\alpha-\beta} c \int_{-\infty}^{\infty}|\exp (-i u)-1||u|^{-1-\alpha+\beta} d u .
\end{aligned}
$$

(Proof. The second order difference is dominated by

$$
c \int_{-\infty}^{\infty}|\exp (i u h)-1| \cdot|\exp (i u l)-1| \cdot|u|^{-1-\alpha} d u
$$

which is at most equal to

$$
\operatorname{ch}^{\beta} \int_{-\infty}^{\infty}|u|^{\beta}|\exp (i u l)-1| \cdot|u|^{-1-\alpha} d u
$$

now substitute $u / l$ for $u$.)

Two more results about the function $r(t)$ in (5.2) are stated in the following lemmas.

LEMMA 5.1. Let $s$ and $t$ be positive quantities converging to 0 in such a way that $s /(s+t) \rightarrow \sigma>0, t /(s+\tau) \rightarrow \tau>0$; and let $h$ be a nonnegative quantity such that $h /(s+t) \rightarrow \gamma($ finite or infinite); then

$$
(s t)^{-\alpha / 2}[r(s+h)+r(t+h)-r(s+t+h)-r(h)]
$$

converges to 0 if $\gamma=\infty$, and to

$$
c(\sigma \tau)^{-\alpha / 2} \int_{-\infty}^{\infty} \exp [-i u(\sigma+\gamma)](\exp (i u \sigma)-1)(\exp (-i u \tau)-1)|u|^{-1-\alpha} d u
$$

if $\gamma$ is finite.

Proof. Write (5.6) in spectral form, changing the variable of integration from $u$ to $u /(s+t)$ :

$$
\begin{aligned}
c \int_{-\infty}^{\infty} \exp [i u(s+h) /(s+t)](\exp [i u t /(s+t)]-1) \\
\cdot(\exp [-i u s /(s+t)]-1) \frac{d u}{s+t+|u|^{1+\alpha}}\left(\frac{s+t}{s} \cdot \frac{s+t}{t}\right)^{\alpha / 2} .
\end{aligned}
$$

The last factor converges to $(\sigma \tau)^{-\alpha / 2}$. For $\varepsilon>0$, the integral over the portion $|u|<\varepsilon$ is at most equal to

$$
\frac{t}{s+t} \cdot \frac{s}{s+t} \int_{-\varepsilon}^{\varepsilon} \frac{u^{2} d u}{s+t+|u|^{1+\alpha}}<\varepsilon^{2-\alpha}
$$


This is negligibly small with $\varepsilon$; therefore, in order to complete the proof, we shall evaluate the limit of the integral over $|u| \geqq \varepsilon$.

The function

$$
(\exp [i u s /(s+t)]-1)(\exp [-i u t /(s+t)]-1) /\left(s+t+|u|^{1+\alpha}\right)
$$

converges, for $u \neq 0$, to

$$
(\exp (i u \sigma)-1)(\exp (-i u \tau)-1)|u|^{-1-\alpha} ;
$$

furthermore, the convergence also holds in the mean of order 1 on the domain $|u| \geqq \varepsilon$; therefore, we are permitted to replace the function (5.9) by its limit (5.10) before taking the limit of the integral (5.8) over the domain $|u| \geqq \varepsilon$. The assertion of the lemma follows: for $\gamma=\infty$ from the Riemann-Lebesgue lemma, and for $\gamma<\infty$, from the continuity of the Fourier transform of an integrable function.

LEMMA 5.2. Let $X(t), 0 \leqq t \leqq t$, be a process with the covariance function (5.2); then the determinant of the covariance matrix of the standardized random variables

$$
\frac{X\left(t_{j}\right)-X\left(t_{j+1}\right)}{\left[2\left(1-r\left(t_{j+1}-t_{j}\right)\right)\right]^{1 / 2}}, \quad X\left(t_{n}\right), \quad(j=1, \ldots, n-1)
$$

is bounded away from 0 on the subset of the cube in $n$-space:

$$
\left\{\left(t_{1}, \ldots, t_{n}\right): 0 \leqq t_{1}<\cdots<t_{n} \leqq 1\right\} .
$$

Proof. The determinant is strictly positive on the set (5.12): if it were not so, then there would be a linear relation among the random variables $X\left(t_{j}\right)$, contradicting the absolute continuity of the spectrum. To prove that the determinant is bounded away from 0 we shall show that if it converges to a limit along a sequence converging to a point on the boundary of (5.12) outside the set, then the limit is positive. Such boundary points are those with $0 \leqq t_{1} \leqq \cdots \leqq t_{n} \leqq 1$ with at least one equality between $t$ 's.

Our goal is equivalent to proving: if the joint distribution of the random variables (5.11) converges to a limiting distribution (necessarily Gaussian) as one or more of the differences $t_{j+1}-t_{j}$ tends to 0 , then the limiting distribution is nonsingular. From a sequence in (5.12) converging to the boundary we can, by compactness, extract a subsequence for which the points $t_{1}, \ldots, t_{n}$ and the ratios $\left(t_{j+1}-t_{j}\right) /\left(t_{k+1}-t_{k}\right)$ all converge to limits, finite or infinite; therefore, we shall assume that the original sequence also has this property. As a point in (5.12) moves toward the boundary the differences $t_{+1}-t_{j}$ may be of varying order of magnitude. By (5.3) the denominator in (5.11) is asymptotic to a constant multiple of $\left|t_{j+1}-t_{j}\right|^{\alpha / 2}$ as the difference $t_{j+1}-t_{j}$ tends to 0 . Each standardized increment in (5.11) for which $t_{j+1}-t_{j} \rightarrow 0$ has limiting correlation 0 with every increment for which $t_{j+1}-t_{j}$ is bounded away from 0 , and with $X\left(t_{n}\right)$; this follows from (5.4) with $\beta$ selected so that $\alpha / 2<\beta<1$. If $t_{j+1}-t_{j}$ and $t_{k+1}-t_{k}$ both tend to 0 but the ratio of the former to the latter tends to 0 , then the corresponding standardized 
increments have the limiting covariance 0 ; this follows from (5.3) and (5.5) with $h=t_{j+1}-t_{j}, l=t_{k+1}-t_{k}, \alpha / 2<\beta<1$. The final case to consider is when two differences tend to 0 and their ratio converges to a positive finite limit. Suppose $t_{j+1}<t_{k}$. If $\left(t_{k}-t_{j+1}\right) /\left(t_{j+1}-t_{j}\right) \rightarrow \infty$, the limiting correlation of the corresponding increments (5.11) is 0 ; this follows from Lemma 5.1 with $\gamma=\infty, s=t_{j+1}-t_{j}, h=t_{k}-t_{j+1}$. If $\left(t_{k}-t_{j+1}\right) /\left(t_{j+1}-t_{j}\right)$ has a finite limit, the corresponding covariance converges to a limit determined by Lemma 5.1. It is only in this last case that nonzero limiting covariance arises among the increments in (5.1); we shall show that the limiting joint distribution of such increments is nonsingular.

For the proof it is sufficient to show: if $t_{1}<t_{2}<\cdots<t_{n}, t_{n}-t_{1} \rightarrow 0$, and the limits

$$
\tau_{j}=\lim \left(t_{j+1}-t_{1}\right) /\left(t_{n}-t_{1}\right), \quad j=1, \ldots, n-1,
$$

satisfy $0<\tau_{1}<\cdots<\tau_{n-1}$, then the limiting distribution of the increments (5.11) is nonsingular. By Lemma 5.1 the limit of the correlation of $X\left(t_{j}\right)-X\left(t_{j+1}\right)$ and $X\left(t_{k}\right)-X\left(t_{k+1}\right)$ is

$$
\frac{\int_{-\infty}^{\infty}\left(\exp \left(i u \tau_{j+1}\right)-\exp \left(i u \tau_{j}\right)\right)\left(\exp \left(-i u \tau_{k+t}\right)-\exp \left(-i u \tau_{k}\right)\right)|u|^{-1-\alpha} d u}{\int_{-\infty}^{\infty}|1-\exp (i u)|^{2}|u|^{-1-\alpha} d u \cdot\left|\tau_{j+1}-\tau_{j}\right|^{\alpha / 2}\left|t_{k+1}-\tau_{k}\right|^{\alpha / 2}} .
$$

We claim that the limit correlation matrix is nonsingular. Let $v_{1}, \ldots, v_{n-1}$ be complex numbers such that

$$
\int_{-\infty}^{\infty}\left|\sum_{j=1}^{n-1} v_{j} \frac{e^{i u \tau_{j+1}}-e^{i u \tau},}{\left|\tau_{j+1}-\tau_{j}\right|^{\alpha / 2}}\right|^{2}|u|^{-1-\alpha} d u=0
$$

then, the integrand vanishes for almost all $u$, or, equivalently, the function

$$
\sum_{j=1}^{n-1} v_{j}\left|\tau_{j+1}-\tau_{j}\right|^{-\alpha / 2} \int_{\tau_{j}}^{\tau_{j+1}} \exp (\text { iuy }) d y
$$

vanishes for almost all $u$. The latter is the Fourier transform of the function taking the value $v_{j}\left(\tau_{j+1}-\tau_{j}\right)^{-\alpha / 2}$ on $\left[\tau_{j}, \tau_{j+1}\right]$; thus, the transform vanishes for almost all $u$ only if $v_{j}=0$ for all $j$; therefore the matrix is nonsingular.

It follows from the analysis just completed that the joint limiting distribution of the set (5.11) is a product of nonsingular Gaussian distributions; so it is nonsingular.

The essential results for the particular covariance (5.2) are now extended.

LEMMA 5.3. Let $X(t)$ be a stationary Gaussian process whose spectral distribution $G$ satisfies all the conditions up to and including (5.1); then its covariance satisfies (5.3) and the conclusion of Lemma 5.2 holds.

Proof. Put $2(1-r(t))$ in spectral form as the integral of $\mid 1-\left.\exp ($ iut $)\right|^{2}$, and write $d G(u)$ as

$$
c d u /\left(1+|u|^{1+\alpha}\right)+\left[d G(u)-c d u /\left(1+|u|^{1+\alpha}\right)\right] .
$$


The integral with respect to the second term of this differential is, by condition (5.1) and the inequality $\left|1-e^{i x}\right| \leqq|x|$, of the order $t^{2}$; therefore (5.3) holds.

The decomposition (5.13) implies that the covariance of $X\left(t_{j}\right)-X\left(t_{j+1}\right)$ and $X\left(t_{k}\right)-X\left(t_{k+1}\right)$ differs from that for the particular process with covariance (5.2) by a quantity of the order $\left|t_{j+1}-t_{j}\right| \cdot\left|t_{k+1}-t_{k}\right|$, which is of smaller order than the product of the standard deviations. A similar result holds for the covariance of $X\left(t_{n}\right)$ and an increment $X\left(t_{j}\right)-X\left(t_{j+1}\right)$. It follows that the limiting nonsingularity established in Lemma 5.2 holds also for our process.

Before stating the main theorem, we derive an inequality for the characteristic function of the multivariate Gaussian distribution. Let $\left(X_{1}, \ldots, X_{k}\right)$ be a random vector with a multivariate Gaussian distribution with mean vector 0 and covariance matrix $\left(r_{i j}\right)$ with determinant $\left\|r_{i j}\right\|>0$. (We refer to [4, p. 310] for definitions and fundamentals.) The joint characteristic function of $\left(X_{1}, \ldots, X_{k}\right)$ is given by

$$
E \exp \left[i\left(u_{1} X_{1}+\cdots+u_{k} X_{k}\right)\right]=\exp \left(-\frac{1}{2} \sum_{i, j=1}^{k} r_{i j} u_{i} u_{j}\right)
$$

Let $c_{h}$ be the cofactor of the diagonal element $r_{h h}$ of $\left(r_{i j}\right) ; c_{h}$ is positive because it is a principal subdeterminant of $\left\|r_{i j}\right\|, h=1, \ldots, k$. The conditional distribution of $X_{h}$, given all the $X_{l}, l \neq h$, is a univariate Gaussian distribution with (conditional) mean $E\left(X_{h} \mid X_{l}, l \neq h\right)$ and (conditional) variance $\left\|r_{i j}\right\| / c_{h}$; thus, the conditional characteristic function of $X_{h}$, given $X_{l}, l \neq h$, is given by the formula

$$
E\left(\exp \left[i u X_{h}\right] \mid X_{l}, l \neq h\right)=\exp \left[i u E\left(X_{h} \mid X_{l}, l \neq h\right)-\frac{1}{2} u^{2}\left\|r_{i j}\right\| / c_{h}\right]
$$

The following is a result about positive definite quadratic forms.

LEMMA 5.4. Let $\left(r_{i j}\right)$ be a $k \times k$ nonsingular positive definite symmetric matrix and $c_{h}$ the cofactor of the element $r_{h h}$; then

$$
\sum_{i, j=1}^{k} r_{i j} u_{i} u_{j} \geqq\left\|r_{i j}\right\| \cdot k^{-1} \sum_{j=1}^{k}\left(u_{j}^{2} / c_{j}\right)
$$

for all vectors $\left(u_{1}, \ldots, u_{k}\right)$.

Proof. Let $\left(X_{1}, \ldots, X_{k}\right)$ be a random vector with a multivariate Gaussian distribution with mean vector 0 and covariance matrix $\left(r_{i j}\right)$. Using a standard conditioning process on the joint characteristic function, and the formula (5.15), we obtain

$$
\begin{aligned}
E \exp \left[i \sum_{j} u_{j} X_{j}\right] & =E\left[E\left(\exp \left[i \sum_{j} u_{j} X_{j}\right] \mid X_{l}, l \neq h\right)\right] \\
& =E\left[\exp \left[i \sum_{j \neq h} u_{j} X_{j}\right] E\left(\exp \left[i u_{h} X_{h}\right] \mid X_{l}, l \neq h\right)\right] \\
& =E\left[\exp \left[i \sum_{j \neq h} u_{j} X_{j}-\frac{1}{2} u_{h}^{2}\left\|r_{i j}\right\| / c_{h}+i u_{h} E\left(X_{h} \mid X_{l}, l \neq h\right)\right]\right]
\end{aligned}
$$


The expressions above are, by (5.14), real and positive, and the last is dominated by $\exp \left(-\frac{1}{2} u_{h}^{2}\left\|r_{i j}\right\| / c_{h}\right)$; in other words, it has been shown that

$$
\exp \left(-\frac{1}{2} \sum_{i, j=1}^{k} r_{i j} u_{i} u_{j}\right) \leqq \exp \left(-\frac{1}{2} u_{h}^{2}\left\|r_{i j}\right\| / c_{h}\right), \quad h=1, \ldots, k,
$$

or, equivalently,

$$
\sum_{i, j=1}^{k} r_{i j} u_{i} u_{j} \geqq\left\|r_{i j}\right\| u_{h}^{2} / c_{h}, \quad h=1, \ldots, k .
$$

The left-hand side of the above inequality is independent of $h$; hence, the inequality asserted in the lemma follows by averaging over $h=1, \ldots, k$.

THEOREM 5.1. Under the conditions up to and including (5.1), there is a local time $\phi(x, \omega)$ continuous in $x$ for almost all $\omega$.

Proof. We evaluate the expectation (4.5) for this particular process, showing that the continuity criterion is satisfied. Put $n=2 m$; then $E|\psi(x+h)-\psi(x)|^{n}$ is dominated by

$$
(1 / 2 \pi)^{n} \int_{0}^{1} \cdots \int_{0}^{1} \int_{-\infty}^{\infty} \cdots \int_{-\infty}^{\infty} \prod_{j=1}^{n}\left|1-\exp \left(i u_{j} h\right)\right| E\left[\exp \left(i \sum_{j=1}^{n} u_{j} X\left(t_{j}\right)\right)\right] \prod_{j=1}^{n} d u_{j} d t_{j} .
$$

Let $\delta$ be a positive number less than 1 ; its magnitude will be more exactly determined below. By the inequality $\left|1-e^{i x}\right| \leqq 2|x|^{\delta}$, the above expression is bounded by $h^{n \delta}(1 / \pi)^{n}$ times the integral

$$
\int_{0}^{1} \cdots \int_{0}^{1} \int_{-\infty}^{\infty} \cdots \int_{-\infty}^{\infty} \prod_{j=1}^{n}\left|u_{j}\right|^{\delta} E\left[\exp \left(i \sum_{j=1}^{n} u_{j} X\left(t_{j}\right)\right)\right] \prod_{j=1}^{n} d u_{j} d t_{j}
$$

The continuity condition is satisfied if there is a sufficiently small $\delta>0$ and a sufficiently large $n$ satisfying $n \delta>1$ such that the integral (5.16) is finite.

It will be shown that the integral (5.16) is finite for all $n \geqq 2$ if

$$
\delta<(1 / \alpha)-(1 / 2)
$$

For this purpose integration over the unit cube (with respect to $t_{1}, \ldots, t_{n}$ ) may be replaced by integration over the subset $(5.12)$. Put

$$
\sigma_{j}^{2}=2\left(1-r\left(t_{j+1}-t_{j}\right)\right), \quad j=1, \ldots, n-1,
$$

and change variables in the inner multiple integral:

$$
u_{1}=v_{1} / \sigma_{1}, \quad u_{j}=v_{j} / \sigma_{j}-v_{j-1} / \sigma_{j-1}, \quad j=2, \ldots, n .
$$

Under this transformation the integrand takes the form

$$
\begin{aligned}
&\left|v_{1} / \sigma_{1}\right|^{\delta} \prod_{j=1}^{n-1} \sigma_{j}^{-1} \prod_{j=2}^{n}\left|v_{j} \sigma_{j}-v_{j-1} / \sigma_{j-1}\right|^{\delta} \\
& \cdot E\left\{\exp \left[i \sum_{j=1}^{n-1} v_{j} \sigma_{j}^{-1}\left(X\left(t_{j}\right)-X\left(t_{j+1}\right)\right)+v_{n} X\left(t_{n}\right)\right]\right\}
\end{aligned}
$$


By the inequality $|x-y|^{\delta} \leqq|x|^{\delta}+|y|^{\delta}$, this is dominated by a linear combination of terms of the form

$$
\prod_{j=1}^{n-1} \sigma_{j}^{-1-2 \delta} \prod_{j=1}^{n}\left|v_{j}\right|^{\theta_{j} \delta} \exp \left[-\frac{1}{2} \sum_{j, k=1}^{n} r_{i j} v_{i} v_{j}\right]
$$

where the variables $\theta_{j}$ assume at most the values 0,1 and 2 , and where $\left(r_{i j}\right)$ is the covariance matrix of the random variables (5.11). Apply the inequality of Lemma 5.4 and integrate over $v_{1}, \ldots, v_{n}$ : the integral is dominated by a constant multiple of

$$
\prod_{j=1}^{n-1} \sigma_{j}^{-1-2 \delta} \prod_{h=1}^{n}\left(2 n c_{h} /\left\|r_{i j}\right\|\right)^{\left(\theta_{h} \delta+1\right) / 2} ;
$$

indeed, when the quadratic form in the exponent is replaced by the weighted sum of squares, the integral becomes a product of $n$ (elementary) integrals. The principal subdeterminants $c_{h}$ are all bounded by 1 ; by Lemma $5.3,\left\|r_{i j}\right\|$ is bounded away from 0 on the domain of integration. It follows that the function (5.18) is bounded by a constant multiple of $\prod_{j=1}^{n-1} \sigma_{j}^{-1-2 \delta}$. The latter is integrable over (5.12) if $\delta$ satisfies (5.17). This completes the proof.

6. Local times and first passage times. We use the local time to derive some apparently new inequalities for first passage times of stationary Gaussian processes.

Lemma 6.1. Let $X(t, \omega), 0 \leqq t \leqq 1$, be a separable stationary Gaussian process with local time $\phi(x, \omega)$ such that $X(t, \omega)$ and $\phi(x, \omega)$ are continuous in $t$ and $x$, respectively, for almost all $\omega$. The probability that $x$ is not in the range of $X(\cdot, \omega)$ is less than or equal to the probability that $\phi(x, \omega)$ is equal to 0 .

Proof. The complement of the range of a continuous function on a compact set is open; thus, if $x$ is not in the range of $X(\cdot, \omega)$, the latter spends no time in some neighborhood of $x$; hence, the local time must vanish almost everywhere in this neighborhood, and, as a continuous function, the local time vanishes at $x$ itself.

Corollary 6.1. Under the hypothesis of the lemma and the condition (3.2) the probability that $x$ is not in the range of the sample function is not greater than

$$
\int_{0}^{1} \int_{0}^{1}\left(1-r^{2}\right)^{-1 / 2} \exp \left(r x^{2} / 1+r\right) d s d t-1
$$

where $r=r(s-t)$.

Proof. We use a variant of the Chebyshev inequality employed by Cramér [5]: For any random variable $Y$ with finite variance, we have $P\{Y=0\} \leqq \operatorname{Var}(Y) /(E Y)^{2}$. We choose the particular version $\psi(x, w)$ of $\phi(x, w)$ as constructed in Theorem 4.1, and put $Y=\psi(x, w)$ :

$$
\begin{aligned}
E \psi(x) & =\exp \left[-\left(x^{2} / 2\right)\right] /(2 \pi)^{1 / 2}, \\
E \psi^{2}(x) & =(1 / 2 \pi) \int_{0}^{1} \int_{0}^{1}\left(1-r^{2}\right)^{-1 / 2} \exp \left[-x^{2} /(1+r)\right] d s d t,
\end{aligned}
$$

where $r=r(s-t)$. Direct substitution in the above inequality yields the bound (6.1). 
In view of the closing remarks of $\S 4$, we note that Lemma 6.1 and Corollary 6.1 can be extended from a process on the unit interval to one on an arbitrary interval $[0, t]$ : the domain of integration in $(6.1)$ has to be changed to the square $[0, t] \times$ $[0, t]$, and the integral divided by $t^{2}$. As an application, we obtain an upper bound on the probability that the process does not assume the value 0 anywhere in $[0, t]$ :

$$
t^{-2} \int_{0}^{t} \int_{0}^{t}\left[\left(1-r^{2}\left(s_{1}-s_{2}\right)\right)^{-1 / 2}-1\right] d s_{1} d s_{2}
$$

This is certainly not greater than

$$
(2 / t) \int_{0}^{t}\left[\left(1-r^{2}(s)\right)^{-1 / 2}-1\right] d s .
$$

If the process is ergodic, then, as a consequence of the ergodic theorem, the process must eventually pass through 0 ; thus, the probability that there are no zeros in $[0, t]$ converges to 0 as $t \rightarrow \infty$. In the ergodic case, the bound (6.2) converges to 0 as $t \rightarrow \infty$. To prove this, we recall our assumption that $r(t)$ is bounded away from 1 outside a neighborhood of $t=0$; then, by the law of the mean, $\left(1-r^{2}\right)^{-1 / 2}-1$ is bounded by a constant multiple of $r^{2}$ for all $t>0$ outside a neighborhood of the origin; therefore, the convergence of the expression (6.2) to 0 is implied by the condition

$$
\lim _{t \rightarrow \infty} t^{-1} \int_{0}^{t} r^{2}(s) d s=0 .
$$

This is exactly the condition for ergodicity.

\section{Multiplicity of the values of the sample functions.}

THEOREM 7.1. Let $X(t, \omega), 0 \leqq t \leqq 1$, be a separable stationary Gaussian process with continuous sample functions and with a continuous local time. Then the set of values $x$ where $X(\cdot, \omega)$ crosses $x$ at most finitely often is of category 1 in the range of $X(\cdot, \omega)$ for almost all $\omega$.

Proof. For each $n$, no two of the values $X\left(k 2^{-n}, \omega\right), k=0,1, \ldots, 2^{n}$, are the same for almost all $\omega$; thus, the hypothesis of Theorem 2.1 is fulfilled.

8. The values of unbounded Gaussian sample functions. There is an inverse relationship between the regularity of the sample functions of Gaussian processes and the regularity of the sample functions of their local times: the former is enhanced by the quick approach of $r(t)$ to 1 for $t \rightarrow 0$, and the latter by the slow approach. In this section it will be shown that the local time is an analytic function if $r$ approaches 1 slowly enough; in this case, the sample functions of the Gaussian process are not only unbounded but even spend positive time in every set of positive Lebesgue measure (cf. §1).

There are well-known results relating the analyticity of a characteristic function to the rate of decrease of the tails of the corresponding distribution function [10]. 
These results can also be derived in the other direction: If the characteristic function tends to 0 rapidly enough at infinity, then there is a strip in the complex plane containing the real line such that the density of the distribution function exists and may be extended to an analytic function in the strip.

LEMMA 8.1. Let $\hat{\phi}(u)$ be the characteristic function of a distribution function $F(x)$ such that

$$
\int_{0}^{\infty} \exp (u b)|\hat{\phi}(u)| d u<\infty
$$

for some $b>0$. Then $F(x)$ has a density function $\phi(x)$ which may be extended to a function $\phi(z)$ of a complex variable $z$, analytic in the strip $|\operatorname{Im} z|<b$.

Proof. The proof is analogous to the one given in [4, p. 176], so that we omit the details. We formally define the function $\phi(z), z$ complex, as

$$
\phi(z)=\frac{1}{2 \pi} \int_{-\infty}^{\infty} \exp (i z u) \hat{\phi}(u) d u .
$$

Condition (8.1) guarantees the absolute convergence of the integral, the expansion in power series of the exponential and term by term integration, and analytic continuation, all in the infinite strip $|\operatorname{Im} z|<b$. We also use the fact that

$$
|\hat{\phi}(u)|=|\hat{\phi}(-u)| \text {. }
$$

The condition (8.1) can be put in a form more suitable for our purposes:

LEMMA 8.2. The condition (8.1) is satisfied if

$$
\int_{0}^{\infty} \exp (4 u b)|\hat{\phi}(u)|^{2} d u<\infty .
$$

Proof. Write the integral in (8.1) as

$$
\int_{0}^{\infty}(\exp (2 u b)|\hat{\phi}(u)|)(\exp (-u b)) d u
$$

and apply the Cauchy-Schwarz inequality.

THEOREM 8.1. Let $X(t, \omega), 0 \leqq t \leqq 1$, be a stationary Gaussian process whose correlation function $r(t)$ satisfies the condition

$$
\liminf _{t \rightarrow \infty}|\log | t||(1-r(t))>0 .
$$

Then, for almost all $\omega$, there exists a version $\phi(x, \omega)$ of the local time whose sample functions have analytic extensions to a strip in the complex plane containing the real line.

Proof. Let $\hat{\phi}(u, \omega)$ be the characteristic function of $F(x, \omega)$ for each $\omega$. We shall prove that for some $b>0, \int_{0}^{+\infty} \exp (u b)|\hat{\phi}(u, \omega)|^{2} d u<\infty$ for almost all $\omega$. (Lemmas 
8.1 and 8.2 then imply the conclusion of the theorem.) It suffices to show that the expected value of the above integral is finite: and the measurability of $X(t, \omega)$ in $(t, \omega)$ implies the sufficiency of showing that

$$
\int_{0}^{\infty} \exp (u b) E|\hat{\phi}(u)|^{2} d u<\infty
$$

By definition, the integral in (8.4) is equal to

$$
\int_{0}^{\infty} \exp (u b) \int_{0}^{1} \int_{0}^{1} E \exp (i u[X(t)-X(s)]) d s d t d u
$$

which is equal to

$$
\int_{0}^{\infty} \exp (u b) \int_{0}^{1} \int_{0}^{1} \exp \left(-u^{2}[1-r(s-t)]\right) d s d t d u .
$$

Interchange the order of integration and apply the formula

$$
\int_{-\infty}^{\infty} \exp \left[u b-u^{2}(1-r)\right] d u=[\pi /(1-r)]^{1 / 2} \exp \left[b^{2} / 4(1-r)\right]:
$$

the integral (8.5) is dominated by

$$
\begin{aligned}
(\pi)^{1 / 2} \int_{0}^{1} \int_{0}^{1}(1-r(s-t))^{-1 / 2} & \exp \left[b^{2} / 4(1-r(s-t))\right] d s d t \\
& =2(\pi)^{-1 / 2} \int_{0}^{1}(1-t)(1-r(t))^{1 / 2} \exp \left[b^{2} / 4(1-r(t))\right] d t \\
& \leqq 2(\pi)^{1 / 2} \int_{0}^{1}(1-r)^{-1 / 2} \exp \left[b^{2} / 4(1-r(t))\right] d t .
\end{aligned}
$$

The finiteness of the latter integral depends only on the values of $r(t)$ for small $t$; therefore, by condition (8.3) it is implied by the finiteness of the integral

$$
\int_{0}^{1} t^{-b^{2} / B}(-\log |t|)^{1 / 2} d t
$$

where $B=4 \lim \inf _{t \rightarrow 0}-\log |t|(1-r(t))$. This integral is certainly finite if $b^{2}<B$; hence, the condition (8.4) holds for such $b>0$.

A process with an analytical local time has very erratic sample functions: the process spends positive time in every set of positive measure. This is seen as follows. Suppose $A$ is a measurable set. The amount of time spent in $A$ is the integral of the local time over $A$. If the integral is 0 , then the local time must vanish almost everywhere on $A$; if, in addition, $A$ had positive measure, then the local time would vanish on a set of positive measure; hence, as an analytic function, the local time would vanish everywhere. This contradicts the fact that the integral of the local time over the whole line is necessarily 1 ; therefore, we conclude that $A$ cannot have positive measure; therefore, it must have measure 0 . We have shown that if the process spends 0 time in a measurable set, then the set must have Lebesgue measure 0 . 
The results above are valid if $[0,1]$ is replaced by an arbitrary interval; hence, for each nonempty open subinterval $I$ of $[0,1]$ with rational endpoints, there exists an $\omega$-set $N_{I}$ of probability 0 such that $X(t, \omega), t \in I$, spends positive time in every set of positive measure for $\omega$ outside $N_{I}$. It follows that if $\omega$ is outside $\bigcup N_{I}$ (union over all $I$ with rational endpoints), then the inverse image of a set $A$ of positive measure under the function $X(t, \omega), 0 \leqq t \leqq 1$, intersects every $I$ with rational endpoints in a set of positive measure. Since every subinterval of $[0,1]$ is a monotone limit of one with rational endpoints, the last result is valid for every interval $I$. We conclude: For almost every sample function, the inverse image of a set of positive measure has an intersection of positive measure with every nonempty open subinterval of $[0,1]$. Since almost every sample function is a measurable function of $t$, we have arrived at a probabilistic proof of the theorem of Carathéodory $[3$, p. 214].

\section{REFERENCES}

1. Ju. K. Beljaev, Continuity and Hölder's conditions for sample functions of stationary Gaussian processes, Proc. Fourth Berkeley Sympos. Math. Stat. and Prob., Vol. 2: Contributions to probability theory, Univ. of California Press, Berkeley, Calif., 1961, pp. 23-33.

2. E. S. Boylan, Local times for a class of Markoff processes, Illinois J. Math. 8 (1964), 19-39.

3. C. Carathéodory, Theory of functions, Vol. 2, 2nd English ed., Chelsea, New York, 1960.

4. H. Cramér, Mathematical methods of statistics, Princeton Univ. Press, Princeton, N. J., 1946.

5. —_ On the maximum of a normal stationary stochastic process, Bull. Amer. Math. Soc. 68 (1962), 512-516.

6. H. Cramér and M. R. Leadbetter, Stationary and related stochastic processes: Sample function properties and their applications, Wiley, New York, 1967.

7. J. L. Doob, Stochastic processes, Wiley, New York, 1953.

8. K. Itô and H. P. McKean, Jr., Diffusion processes and their sample paths, Springer-Verlag, Berlin, 1965.

9. P. Lévy, Sur certains processus stochastiques homogènes, Compositio Math. 7 (1939), 283-339.

10. E. Lukacs, Characteristic functions, Griffin's Statistical Monographs \& Courses, No. 5, Hafner, New York, 1960.

11. H. Trotter, A property of Brownian motion paths, Illinois J. Math. 2 (1958), 425-433.

12. J. Yeh, Differentiability of sample functions in Gaussian processes, Proc. Amer. Math. Soc. 18 (1967), 105-108.

13. N. D. Ylvisaker, The expected number of zeros of a stationary Gaussian process, Ann. Math. Statist. 36 (1965), 1043-1046.

NeW YoRK UNIVERSITY, NeW YoRK, New YorK 